\title{
Effect of Build Orientation on the Mechanical Properties and Fracture Behavior of ABS Produced by Fused Deposition Modeling
}

\author{
F. Akasheh ${ }^{1}$, A. Rochester ${ }^{1}$ and H. Aglan ${ }^{1}$ \\ 1. Mechanical Engineering Department, Tuskegee University, Tuskegee, Alabama, USA
}

Additive manufacturing (AM), commonly known as 3D printing, is a recent development in manufacturing processes whereby parts are built incrementally, layer by layer adding material where needed based on slices extracted from a CAD model [1]. The new approach offers significant advantages over conventional manufacturing, including unlimited complexity, higher performance parts, improved sustainability, reduction of assembly and mass customization. Nevertheless, parts made by AM suffer from high anisotropy and inherent weakness in mechanical properties especially in the plane of the layers compared to parts manufactured by conventional methods $[2,3,4]$.

In this work, we study the effect of build orientation on the fracture behavior of polymers made by filament-based extrusion (better known as fused deposition modeling), which is the most versatile and wide spread AM process for polymers. Build orientation is one of the most important process parameters which affect the mechanical behavior of parts built by this process and has been studied under tensile, flexure, compression and impact loading, but not using notched tensile test $[2,3,4]$. Three different build orientations were studied: flat, upright and at $45^{\circ}$-inclined, Figure 1. In all cases, ABS samples with rectangular cross section were built using Stratasys uPrint SE plus FDM printer. The samples had a length of 4", thickness of 0.16 " and width of 0.5 ". After printing, the samples were notched as shown, and tested under uniaxial tension. The different orientations result is different relationships between the loading axis and the plane of the layers. The flat build orientation divides the crack, the $45^{\circ}$ inclined build orientation deflects the crack, while the upright build orientation allows easy interlayer crack propagation. The tensile loading was performed on MTS Sintech 5/D materials testing system equipped with a $5000 \mathrm{lb}$. load cell with cross head speed of $0.5 \mathrm{~mm} / \mathrm{min}$. The fracture surface was also examined to correlate the mechanical testing results and the fractorgraphy results, which were performed using Hitachi S-3400 N Scanning Electron Microscope operated in variable pressure vacuum mode (40 Pa) with an accelerating voltage of $10 \mathrm{kV}$ at $500 \mu \mathrm{m}$ level.

Figure 1 compares the nominal stress-strain curves for the three build orientations, while Figure 2 shows the corresponding fracture morphology. The upright case has the least strength and toughness while the $45^{\circ}$-inclined sample has the highest. The interlayer crack propagation in the upright case has only to cut through the interface between the fused beads which offers the least resistance to crack propagation compared to cutting through the beads (deposited filaments). In the case of the $45^{\circ}$-inclined sample, the crack path is diverted to follow a longer path explaining the higher toughness and ductility as well as the higher strength, Figure 2.c3. The flat build orientation leads to strength and toughness in between the two previous cases. Instead of traveling interlayer or along the longer $45^{\circ}$ path, the crack cuts through the beads normally, facing higher resistant to propagation. The SEM images correlate well with the above observations. Figures 2.a are typical of ductile fracture was shown by the sharp ridges, while Figures 2.b shows a fracture between layers with no significant deformation preceding failure. Finally Figures 2.c suggest the largest amount of shear plastic deformation preceding the fracture. In conclusion, the study shows that the strength and toughness of polymeric parts produced by fused deposition modeling can be controlled by controlling the crack propagation path through the build orientation [5]. 


\section{References:}

[1] I. Gibson, D.W. Rosen, B.Stucker, “Additive manufacturing Technologies”, (Springer, NY) 2010.

[2] S.-H. Ahn et al, Rapid Prototyping Journal 8 (2002), p. 248.

[3] A. R. Torrado et al, Additive Manufacturing 6 (2015), p. 16.

[4] A. K. Sood, R. K. Ohdar, S. S. Mahapatra, Materials and Design 31 (2010), p. 287.

[5] The authors would like to acknowledge the support of Chevron.

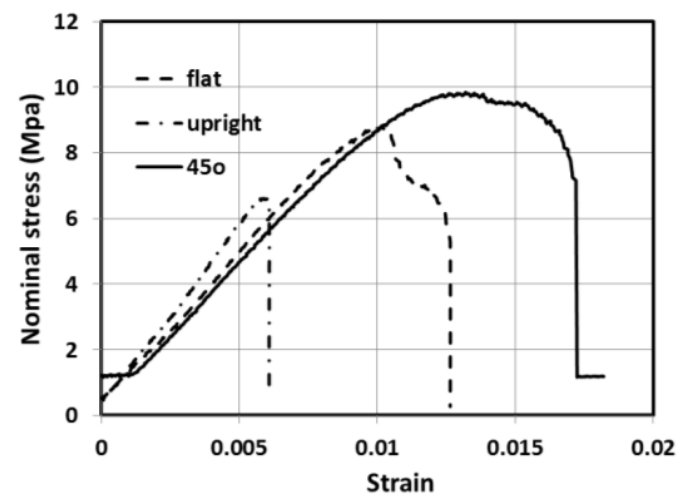

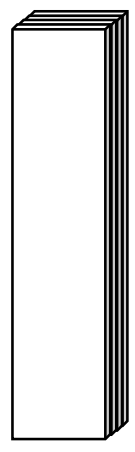

(a) flat

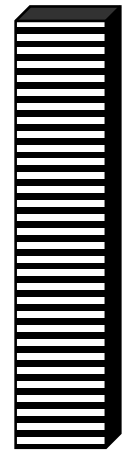

( b)upright

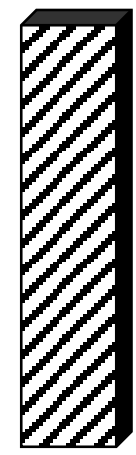

(c) $45^{\circ}$

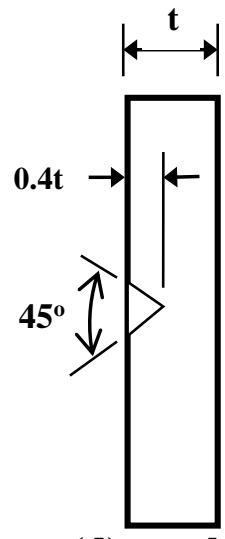

(d) notch

Figure 1. Comparison of the notched tensile stress-strain curve for the different build orientations. The hatch lines indicate the build plane, i.e., the plane of the layers in which the samples were built.

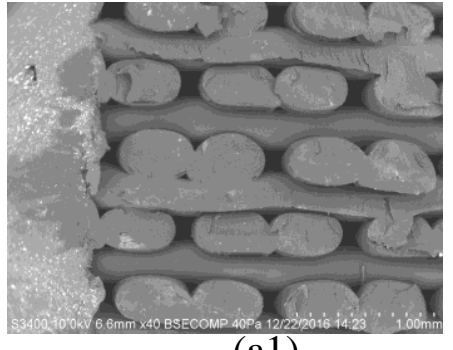

(a1)

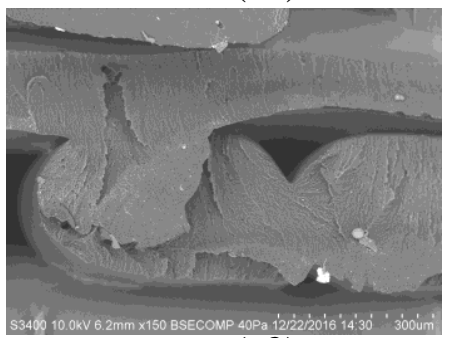

(a2)

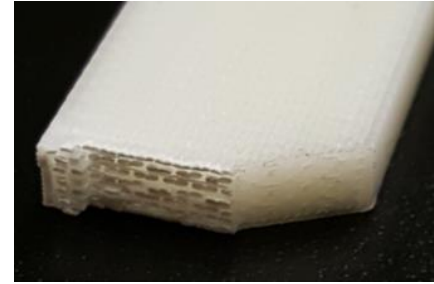

(a3)

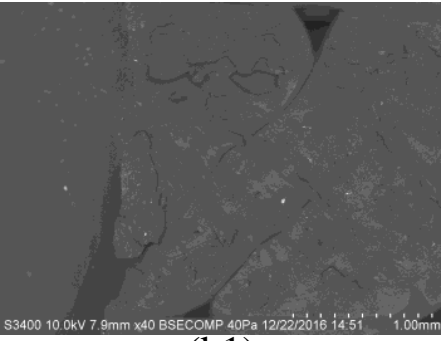

(b1)

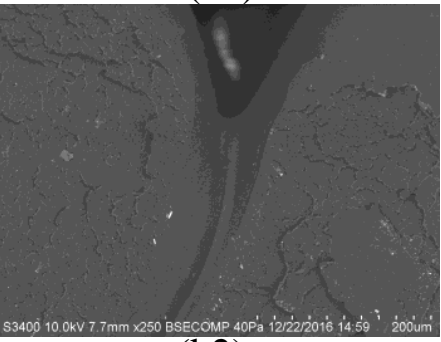

(b2)

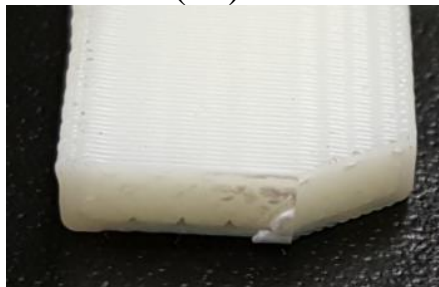

(b3)

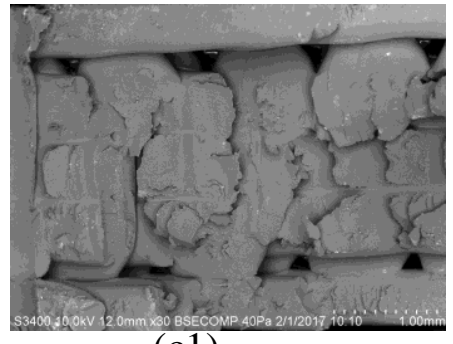

(c1)

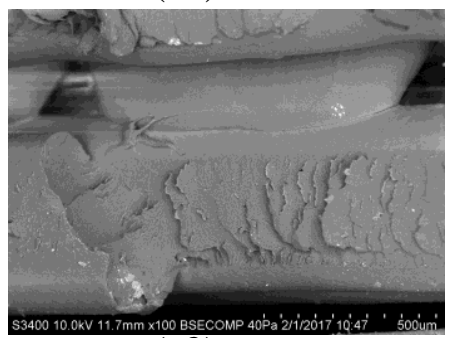

(c2)

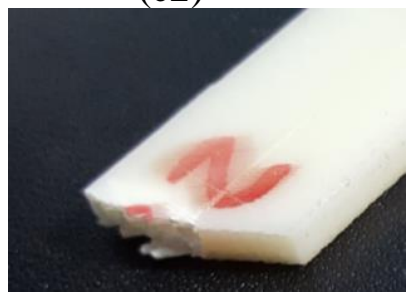

(c3)

Figure 2. SEM and optical images for the fracture surface of the samples. Image sets a, b, and $\mathrm{c}$ are for flat, upright and $45^{\circ}$-inclided build orientations, respectively. Sets 1, 2, 3 indicate low resolution SEM, high resolution SEM and optical images, respectively 